\title{
Author Correction: Distinct thalamocortical network dynamics are associated with the pathophysiology of chronic low back pain
}

Yiheng Tu D, Zening Fu, Cuiping Mao, Maryam Falahpour, Randy L. Gollub (D), Joel Park (D), Georgia Wilson, Vitaly Napadow, Jessica Gerber, Suk-Tak Chan, Robert R. Edwards, Ted J. Kaptchuk (D, Thomas Liu, Vince Calhoun, Bruce Rosen \& Jian Kong (i)

Correction to: Nature Communications https://doi.org/10.1038/s41467-020-17788-z, published online 7 August 2020.

The original version of this Article contained an error in the author affiliation.

Affiliation 4 incorrectly read 'Department of Medical Imaging, First Affiliated Hospital of Xi'An Jiao Tong University College of Medicine, Xi'an, Shaan'Xi, China'.

This has now been corrected in both the PDF and HTML versions of the Article.

Published online: 25 August 2020

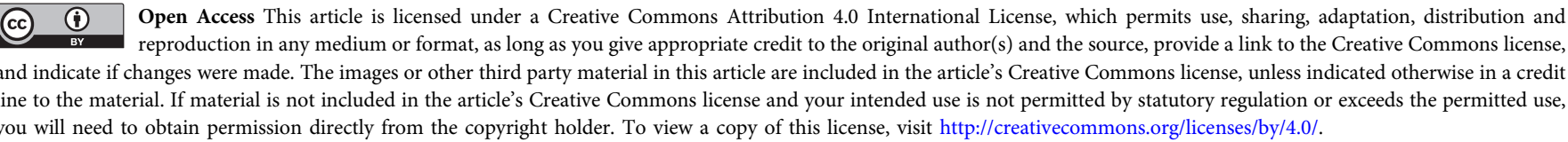

(c) The Author(s) 2020 